\title{
A Mountain Pass-type Theorem for Vector-valued Functions
}

\author{
Ewa M. Bednarczuk • Enrico Miglierina • Elena Molho
}

Received: 7 October 2010 / Accepted: 27 April 2011 /

Published online: 26 May 2011

(C) Springer Science+Business Media B.V. 2011

\begin{abstract}
The mountain pass theorem for scalar functionals is a fundamental result of the minimax methods in variational analysis. In this work we extend this theorem to the class of $\mathcal{C}^{1}$ functions $f: \mathbb{R}^{n} \rightarrow \mathbb{R}^{m}$, where the image space is ordered by the nonnegative orthant $\mathbb{R}_{+}^{m}$. Under suitable geometrical assumptions, we prove the existence of a critical point of $f$ and we localize this point as a solution of a minimax problem. We remark that the considered minimax problem consists of an inner vector maximization problem and of an outer set-valued minimization problem. To deal with the outer set-valued problem we use an ordering relation among subsets of $\mathbb{R}^{m}$ introduced by Kuroiwa. In order to prove our result, we develop an Ekelandtype principle for set-valued maps and we extensively use the notion of vector pseudogradient.
\end{abstract}

Enrico Miglierina and Elena Molho were partially supported by Ministerio de Ciencia y Innovacion (Spain) under project MTM 2009-09493.

E. M. Bednarczuk

Systems Research Institute, Polish Academy of Sciences, ul. Newelska 6, 01-447 Warszawa, Poland

e-mail: Ewa.Bednarczuk@ibspan.waw.pl

E. M. Bednarczuk

Cardinal Stefan Wyszynski University, ul. Dewajtis 5, 01-815 Warszawa, Poland

E. Miglierina

Dipartimento di Economia, Università dell'Insubria, Via Monte Generoso 71,

21100 Varese, Italy

e-mail: enrico.miglierina@uninsubria.it

E. Molho $(\bowtie)$

Dipartimento di Economia Politica e Metodi Quantitativi, via San Felice 5,

27100 Pavia, Italy

e-mail: molhoe@eco.unipv.it 
Keywords Minimax $\cdot$ Ekeland's principle $\cdot$ Vector optimization • Set-valued optimization

Mathematics Subject Classifications (2010) $49 \mathrm{~J} 53$ • 49K35 • 90C29

\section{Introduction}

Minimax methods constitute a fundamental topic in modern critical point theory for real-valued functionals. For a real-valued functional $f$, the scalar mountain pass theorem ensures the existence of a critical point $x_{0}$ such that

$$
f\left(x_{0}\right)=\inf _{K \in \Gamma} \sup _{x \in K} f(x),
$$

where $\Gamma$ is a given family of sets.

The proofs of the scalar mountain pass theorem are based either on a deformation lemma on the level sets of the function $f$ or on Ekeland's variational principle. An appropriate compactness condition is then used to prove the existence of a critical point at the minimax level. For an extensive survey on minimax methods and on mountain pass theorems for scalar functionals see, e.g., $[5,14,26]$ and the references therein.

The aim of this paper is to study critical points for vector-valued functions via minimax approach.

In this direction the pioneering works by Smale [23-25] paved the way for a new approach to general equilibrium models in economic theory under differentiability assumptions. In [22], Smale outlined an extension of Morse theory to vector-valued functions. In this vein, a classification of critical points for functions taking values in partially ordered vector spaces was recently given in [21].

Two distinct results can be found in the recent literature about minimax techniques for the study of the critical points of vector-valued functions. In [6] a deformation lemma on the level sets of the vector-valued function $f$ is used, while in [19] Ekeland's principle is applied to a suitable scalarization of the vector-valued function $f$.

The aim of the present work is not only to obtain an existence result for critical points in the vector-valued case, where a point is critical if it satisfies the usual first order optimality conditions for the classical Pareto optimization problem, but also to localize these points as limits of solutions of appropriate minimax problems.

Here we study a special formulation of the minimax problem for the vector-valued case. The inner maximization problem consists of a classical Paretian maximization of the vector-valued function $f$. Since the optimal value map of the inner problem is a set-valued map, we are naturally led to investigating the outer minimization problem within the framework of set-valued optimization.

Let $f \in \mathcal{C}^{1}\left(\mathbb{R}^{n}, \mathbb{R}^{m}\right)$. Let us fix two points $x_{1}, x_{2} \in \mathbb{R}^{n}$ such that $x_{1} \neq x_{2}$ and consider the family $\Gamma$ of all compact and connected subsets of $\mathbb{R}^{n}$ containing both $x_{1}$ and $x_{2}$,

$$
\Gamma=\left\{K \subset \mathbb{R}^{n}: K \text { is compact, connected and } x_{1}, x_{2} \in K\right\}
$$


For any $K \in \Gamma$, the inner vector maximization problem

$$
\mathcal{V}-\max _{x \in K} f(x),
$$

consists of the maximization of $f$ with respect to the Pareto order $\leqq$ induced on $\mathbb{R}^{m}$ by the cone $\mathbb{R}_{+}^{m}$. For the set-valued map $\Phi: \Gamma \rightrightarrows \mathbb{R}^{m}$ defined by

$$
\Phi(K):=\operatorname{Max} f(K)
$$

we consider the outer set-valued optimization problem

$$
\mathcal{S}-\min _{K \in \Gamma} \Phi(K) .
$$

For this problem we adopt the solution concept introduced in Definition 3.1 below. We study the following minimax problem

$$
\mathcal{S}-\min _{K \in \Gamma} \mathcal{V}-\max _{x \in K} f(x) \text {. }
$$

(MinMax)

It is well known that in the scalar case a solution of the minimax problem is a critical point for the considered function. In the vector case it is interesting to study relationships between the sets that are solutions of the minimax problem (MinMax) and the critical points of the function $f$. We start by investigating relationships between the problem (MinMax) and the first order optimality conditions for vector optimization problems and we prove that a critical point can always be found in the set of solutions to the problem (Min Max). Next, we prove a mountain pass theorem, i.e., we obtain the existence of critical points for $C^{1}$ vector-valued functions as limits of solutions to perturbed minimax problems under a suitable Palais-Smale condition for vector-valued functions.

An important tool in critical point theory are descent directions. In the $C^{1}$ scalar case the notion of pseudogradient defines a regular field of descent directions at the noncritical points. Here, we use a notion of vector pseudogradient for $C^{1}$ vectorvalued functions introduced in [20]. This notion allows us to select appropriate descent directions without any apriori scalarization procedure.

To our knowledge, the present work provides the first attempt at applying Kuroiwa's concepts of minimality of set-valued optimization problems to variational analysis. We derive a suitable Ekeland's variational principle for set-valued optimization problems and we use it to prove the existence of critical points that are explicitly characterized as limits of solutions of perturbed minimax problems.

We deem that the results presented below, even if formulated in the simple setting of an Euclidean space endowed with the componentwise ordering, enjoy the quality of an easy interpretation in terms of vector-valued models in physics and/or in economics.

\section{Notations and Preliminaries}

Let $f=\left(f_{1}, \ldots, f_{m}\right): \mathbb{R}^{n} \rightarrow \mathbb{R}^{m}$ be a function such that $f \in \mathcal{C}^{1}\left(\mathbb{R}^{n}, \mathbb{R}^{m}\right)$. By $f_{i}$ : $\mathbb{R}^{n} \rightarrow \mathbb{R}(i=1, \ldots, m)$ we denote the components of the function $f$. Moreover, we denote by $\nabla f_{i}(x)(i=1, \ldots, m)$ and $\left[f^{\prime}(x)\right]$ respectively, the gradient of the real-valued function $f_{i}$ and the Jacobian matrix of the function $f$. Let $A \subset \mathbb{R}^{n}$ be a nonempty set, we denote by int $A$ the interior of $A$ and by $\operatorname{cl} A$ the closure of $A$. Moreover, we denote by $B_{r}(0)$ the open ball of radius $r$ around 0 . Finally, $\mathbb{R}_{+}^{m}$ denotes the 
nonnegative orthant of $\mathbb{R}^{m}$ given by $\left\{x=\left(x^{1}, \ldots, x^{m}\right) \in \mathbb{R}^{m}: x^{i} \geq 0, i=1, \ldots, m\right\}$, which induces on $\mathbb{R}^{m}$ the Pareto order $\leqq$, defined as follows: for any $x, y \in \mathbb{R}^{m}$,

$$
x \leqq y \Longleftrightarrow y-x \in \mathbb{R}_{+}^{m} .
$$

Let $A \subset \mathbb{R}^{n}$ be a nonempty set. We consider the vector optimization problem

$$
\mathcal{V}-\max _{x \in A} f(x)
$$

where $\mathcal{V}$ - $\max _{x \in A}$ denotes that we consider the maximization with respect to the Pareto order. The solution set $\operatorname{ArgMax}(f, A)$ of the problem $(\mathcal{V}-P)$ is defined by

$$
\operatorname{ArgMax}(f, A):=\left\{x \in A:(f(A)-f(x)) \cap \mathbb{R}_{+}^{m}=\{0\}\right\}
$$

and the set of the maximal values $\operatorname{Max} f(A)$ is defined by

$$
\operatorname{Max} f(A):=f(\operatorname{ArgMax}(f, A)) .
$$

We recall also a weaker notion of solution of problem $(\mathcal{V}-P)$. We introduce the set

$$
\operatorname{ArgWMax}(f, A):=\left\{x \in A:(f(A)-f(x)) \cap \operatorname{int} \mathbb{R}_{+}^{m}=\emptyset\right\}
$$

of the weak solutions of problem $(\mathcal{V}-P)$ and we define the set of weakly maximal points of $f(A)$ as

$$
\text { WMax } f(A):=f(\operatorname{ArgWMax}(f, A)) \text {. }
$$

It is easy to see that

$$
\operatorname{Max} f(A) \subseteq \operatorname{WMax} f(A) .
$$

Moreover, since WMax $f(A)$ is closed whenever $f(A)$ is closed, it holds (see, e.g., [18])

$$
\operatorname{cl}(\operatorname{Max} f(A)) \subseteq \operatorname{WMax} f(A) .
$$

Now, we recall the definition of critical point for a vector-valued function.

Definition 2.1 A point $x \in \mathbb{R}^{n}$ is called critical for the function $f$ if

$$
\left[f^{\prime}(x)\right]\left(\mathbb{R}^{n}\right) \cap\left(-\operatorname{int}\left(\mathbb{R}_{+}^{m}\right)\right)=\emptyset .
$$

Let $K_{f}$ be the set of critical points for $f$.

The following proposition characterizes the critical points for the function $f$.

Proposition 2.2 (see [24]) The point $x$ is a critical point for the function $f\left(x \in K_{f}\right)$ if and only if there exist $m$ real numbers $\lambda_{1}, \ldots ., \lambda_{m}$ such that $\lambda_{i} \geq 0 \forall i=1, \ldots ., m$ and $\sum_{i=1}^{m} \lambda_{i}=1$, satisfying

$$
\sum_{i=1}^{m} \lambda_{i} \nabla f_{i}(x)=0 .
$$

i.e. if and only if the component gradients are positively linearly dependent. 
It is well known that

$$
\operatorname{ArgWMax}\left(f, \mathbb{R}^{n}\right) \subseteq K_{f},
$$

hence we can interpret condition (1) as a first order optimality condition for the vector optimization problem $(\mathcal{V}-P)$ with $A=\mathbb{R}^{n}$.

\subsection{Vector Pseudogradient}

We devote this short subsection to the notion of vector pseudogradient introduced in [20] which plays a key role in our approach. The main motivation to introduce this notion is the need to find a field of descent directions of $f$ with respect to the Pareto order.

First of all we introduce the following function $s_{f}: \mathbb{R}^{n} \rightarrow \mathbb{R}_{+}$defined by

$$
s_{f}(x):=\min \left\{\left\|\sum_{i=1}^{m} \lambda_{i} \nabla f_{i}(x)\right\|: \lambda_{i} \in \mathbb{R}, \lambda_{i} \geq 0, \sum_{i=1}^{m} \lambda_{i}=1\right\} .
$$

It can be shown that $s_{f}$ is a continuous function on the whole space $\mathbb{R}^{n}$ (Lemma 5.1 in [20]). In addition, by Proposition 2.2, the following equivalence holds:

$$
s_{f}(x)=0 \Longleftrightarrow x \in K_{f} .
$$

Now, we can define the vector pseudogradient.

Definition 2.3 A vector $v \in \mathbb{R}^{n}$ is called vector pseudogradient for $f$ at $x$ when

1. $\|v\| \leq 2 s_{f}(x)$,

2. $\left[f^{\prime}(x)\right] v \in-\left[s_{f}(x)\right]^{2} e-\mathbb{R}_{+}^{m}$ where $e=(1, \ldots, 1) \in \operatorname{int}\left(\mathbb{R}_{+}^{m}\right)$.

We denote by $V_{f}(x)$ the set of all vector pseudogradients for $f$ at $x$. In [20] it is proved that $V_{f}(x)$ is nonempty, convex and compact for every $x \in \mathbb{R}^{n}$. Moreover, $x \in K_{f}$ if and only if $V_{f}(x)=\{0\}$. The following remark will be useful in the sequel.

Remark 2.4 By (2), it is easy to see that $x \notin K_{f}$ if and only if $0 \notin V_{f}(x)$.

Let us introduce the following notation. Let $A \subset \mathbb{R}^{n}$ be a nonempty, closed and convex set. By $m(A)$ we denote the element with minimal norm in $A$. Let the function $v: \mathbb{R}^{n} \rightarrow \mathbb{R}^{n}$ be defined as follows

$$
v(x):=m\left(V_{f}(x)\right) .
$$

First, we recall a useful characterization of the element of minimal norm in $V_{f}(x)$.

Proposition 2.5 (see [20], Proposition 3.7) We have that $v(x)=-m(H(x))$, where

$$
H(x)=\left\{\sum_{i=1}^{m} \lambda_{i} \nabla f_{i}(x): \lambda_{i} \in \mathbb{R}, \lambda_{i} \geq 0, \sum_{i=1}^{m} \lambda_{i}=1\right\} .
$$

We remark that $s_{f}(x)=\|v(x)\|$. Finally, we mention the following continuity property of the function $v$. 
Theorem 2.6 The function $v$ is continuous at every $x \in \mathbb{R}^{n}$.

Proof The thesis follows from the continuity of the set-valued map $V_{f}$ (see Lemma 5.2 in [20]) and from the continuity of the minimal selection (see [1]).

\section{A Variational Principle for Set-valued Maps}

Set-valued optimization in partially ordered vector spaces can be viewed as a generalization of vector optimization. In this direction, a common approach is to consider solutions to set-valued optimization problems as the maximal frontier of the union of the images of the feasible points under a given set-valued mapping (see, e.g., [15]). Recently, new solution concepts were proposed in [17]. These new concepts are based on various ordering relations among sets which are compatible with the original order structure in the image space. Such an approach seems to be more suitable from the point of view of providing an insight into the nature of setvalued optimization problems, especially those arising in applications.

Let $X$ be a topological space. Let $Y$ be a linear topological space with the topological dual $Y^{*}$ and let $P$ be a pointed, closed and convex cone in $Y$ such that $P^{*+}=\left\{\psi \in Y^{*}:\langle\psi, z\rangle>0 \forall z \in P \backslash\{0\}\right\}$ is a nonempty set. We recall that the latter assumption is equivalent to the assumption that $P$ is based whenever $Y$ is an Hausdorff locally convex space (see, e.g., [8], Theorem 2.2.12).

Let $\Omega: X \rightrightarrows Y$ be a set-valued map. We consider the following set-valued optimization problem

$$
\mathcal{S}-\min _{x \in X} \Omega(x),
$$

where a solution $\hat{x} \in X$ is defined as follows.

Definition 3.1 (see [17]) A point $\hat{x} \in X$ is a solution of the set-valued optimization problem $(\mathcal{S}-P)$ if for every $x \in X$,

$$
\Omega(x) \subset \Omega(\hat{x})-P \Longrightarrow \Omega(\hat{x}) \subset \Omega(x)-P .
$$

This definition corresponds to the notion of $u$-type minimal solution of problem $(\mathcal{S}-P)$ in [17]. We remark that, in the special case where $\Omega$ is a vector-valued function, the solutions defined above coincide with the elements of $\operatorname{ArgMax}(\Omega, X)$.

Remark 3.2 For any set $A \subset Y$ the set $\operatorname{Max}_{P}(A)$ of maximal elements of $A$ with respect to $P$ is defined as

$$
\operatorname{Max}_{P}(A):=\{a \in A:(a+P) \cap A=\{a\}\} .
$$

If $A$ is a compact set, by Theorem 4.3, ch.2 in [18], the domination property holds, i.e.

$$
A \subset \operatorname{Max}_{P}(A)-P .
$$

Let $A$ and $B$ be two compact subsets of $Y$ such that $A \subset B-P$ and $B \subset A-$ $P$. Then $\operatorname{Max}_{P}(A)=\operatorname{Max}_{P}(B)$ since by the domination property $\operatorname{Max}_{P}(A)-P=$ $\operatorname{Max}_{P}(B)-P$ holds. 
Now we prove a new version of Ekeland's variational principle for set-valued maps. Some results in this vein received attention in the literature (see [2, 3, 913]). In this section, we follow an approach outlined in [10], Section 4. To our knowledge, the paper [10] is the only paper where a variational principle for setvalued maps is proved with the help of a solution concept resulting from an ordering relation introduced by [17]. Namely, in [10] the $l$-type order relation is considered. In contrast to that approach here we use a notion of solution of a set-valuedoptimization problem based on the so called $u$-type ordering relation among sets (see [17]). Hence we cannot directly refer the reader to the variational principle in [10], but we have to prove an Ekeland-type principle consistent with the solution concept introduced in Definition 3.1.

In this section we prove a variational principle that ensures the existence of a solution for the problem $(\mathcal{S}-P)$ with the perturbed set-valued map $\Omega_{\varepsilon}$ under boundedness and continuity assumptions of the set-valued map $\Omega$.

In order to prove the aforementioned result we introduce an auxiliary scalar function $l_{\psi}$. For any fixed $\psi \in P^{*+}$, we define the following function

$$
l_{\psi}(x):=\sup _{z \in \Omega(x)}\langle\psi, z\rangle .
$$

The first lemma in this section focuses on some boundedness and continuity properties of $l_{\psi}$. We introduce the following notation

$$
\Omega(X):=\bigcup_{x \in X} \Omega(x)
$$

Moreover, we recall that a set $A \subseteq Y$ is said to be $P$-bounded whenever there exists a bounded set $B \subseteq Y$ such that $A \subseteq B+P$.

Lemma 3.3 Let $X, \Omega, \psi$ and $l_{\psi}$ be defined as above and let $x \in X$.

1. If $\Omega(X)$ is P-bounded, then $l_{\psi}$ is bounded from below.

2. If $\Omega$ is lower semicontinuous at $x$, then $l_{\psi}$ is lower semicontinuous at $x$.

Proof We prove the two thesis separately.

1. It follows directly from the definition of $P$-boundedness.

2. By Proposition 2, Ch.9 in [7], we have that

$$
-l_{\psi}(x)=\inf _{z \in \Omega(x)}\langle-\psi, z\rangle
$$

is upper semicontinuous at $x$, hence the thesis follows immediately.

The second lemma is a technical result that establishes a sort of monotonicity property of the set-valued map $\Omega$ with respect to the behavior of the auxiliary function $l_{\psi}$.

Lemma 3.4 Let $X, \Omega, \psi$ and $l_{\psi}$ be defined as above and let $p_{0} \in P \backslash\{0\}$ be a fixed vector. If

$$
l_{\psi}\left(x^{\prime}\right)<l_{\psi}(x)+\alpha\left\langle\psi, p_{0}\right\rangle
$$


for some $\alpha>0$, then

$$
\Omega(x)+\alpha p_{0} \nsubseteq \Omega\left(x^{\prime}\right)-P .
$$

Proof By contradiction let us suppose that

$$
\Omega(x)+\alpha p_{0} \subseteq \Omega\left(x^{\prime}\right)-P .
$$

Then, for every $y \in \Omega(x)$, there exist $y^{\prime} \in \Omega\left(x^{\prime}\right)$ and $p \in P$ such that

$$
y^{\prime}=y+p+\alpha p_{0} .
$$

Hence

$$
\left\langle\psi, y^{\prime}\right\rangle=\langle\psi, y\rangle+\langle\psi, p\rangle+\alpha\left\langle\psi, p_{0}\right\rangle
$$

and therefore

$$
l_{\psi}\left(x^{\prime}\right) \geq\left\langle\psi, y^{\prime}\right\rangle \geq\langle\psi, y\rangle+\alpha\left\langle\psi, p_{0}\right\rangle .
$$

Since $y$ is arbitrary, we obtain

$$
l_{\psi}\left(x^{\prime}\right) \geq l_{\psi}(x)+\alpha\left\langle\psi, p_{0}\right\rangle
$$

a contradiction.

Now we can prove the main result of this section.

Theorem 3.5 (Variational Principle) Let $(X, d)$ be a complete metric space and $Y$ be a locally convex space. Let $P \subset Y$ be a closed convex cone such that $P^{*+} \neq \emptyset$ and $p_{0} \in P \backslash\{0\}$. Let $\Omega$ be a lower semicontinuous set-valued map and let $\Omega(X)$ be a $P$-bounded set. Then for every $\varepsilon>0$ there exists $x_{\varepsilon}$ such that

$$
\Omega(x)+\varepsilon d\left(x_{\varepsilon}, x\right) p_{0} \nsubseteq \subseteq \Omega\left(x_{\varepsilon}\right)-P
$$

for every $x \neq x_{\varepsilon}$.

Proof By separation arguments, we can always find an element $\psi \in P^{*+}$ such that $\left\langle\psi, p_{0}\right\rangle=1$. By Lemma 3.3, $l_{\psi}$ is lower semicontinuous and bounded from below. By Ekeland's Variational Principle, an element $x_{\varepsilon} \in X$ exists such that

$$
l_{\psi}\left(x_{\varepsilon}\right)<l_{\psi}(x)+\varepsilon d\left(x_{\varepsilon}, x\right)
$$

for every $x \neq x_{\varepsilon}$. Now, the thesis follows from Lemma 3.4.

Let us consider the following definition of strict minimizer consistent with the notion of solution given in Definition 3.1.

Definition 3.6 A point $\tilde{x} \in X$ is a strict minimizer (on $X$ ) for the map $\Omega$ if

$$
\Omega(x) \nsubseteq \Omega(\tilde{x})-P
$$

for every $x \in X, x \neq \tilde{x}$.

This notion can be interpreted as an adaptation to our setting of Definition 2.3 in [10] where a different order relation among sets was considered. 
We underline that if $\tilde{x}$ is a strict minimizer for $\Omega$, then it is also a solution in the sense of Definition 3.1 to the set-valued optimization problem $(\mathcal{S}-P)$.

In view of Definition 3.6, the point $x_{\varepsilon}$ in Theorem 3.5 can be considered as a strict minimizer of the set-valued map $\Omega_{\varepsilon}(x)=\Omega(x)+\varepsilon d\left(x, x_{\varepsilon}\right) k_{0}$ obtained by perturbing $\Omega$. Hence, Theorem 3.5 is an existence result for a strict minimizer of the perturbed set-valued map $\Omega_{\varepsilon}$.

\section{Minimax Problem for Vector-valued Functions}

According to the definitions given in Section 1, the minimax problem (MinMax) consists of an inner maximization carried over a feasible region $K$ which plays the role of parameter in the inner problem and the role of variable in the outer minimization problem whose objective function is the optimal value map of the inner problem. Since the inner problem is a classical vector optimization problem, the optimal value is a set given by the whole Pareto maximal frontier. In order to compare the maximal frontiers obtained on different feasible regions $K$, an appropriate partial ordering on the subsets of $\mathbb{R}^{m}$ is introduced. Moreover, we remark that set-valued optimization is naturally involved in our approach since the optimal value map of the inner problem is typically a set-valued map.

As stated in Section 1 , for any $f \in \mathcal{C}^{1}\left(\mathbb{R}^{n}, \mathbb{R}^{m}\right)$ and for any $x_{1}, x_{2} \in \mathbb{R}^{n}$ such that $x_{1} \neq x_{2}$, we consider the family $\Gamma$ of all compact and connected subsets of $\mathbb{R}^{n}$ containing both $x_{1}$ and $x_{2}$,

$$
\Gamma=\left\{K \subset \mathbb{R}^{n}: K \text { is compact, connected and } x_{1}, x_{2} \in K\right\} .
$$

We endow $\Gamma$ with the Hausdorff distance defined as

$$
d_{H}(A, B)=\max \left\{\sup _{x \in A} \inf _{y \in B}\|x-y\|, \sup _{y \in B} \inf _{x \in A}\|x-y\|\right\} \text { for every } A, B \in \Gamma .
$$

Remark 4.1 Since $\mathbb{R}^{n}$ endowed with the usual euclidean metric is complete, by Theorem 4.3.9 in [16], the space of all nonempty compact subsets of $\mathbb{R}^{n}$, endowed with the Hausdorff metric $d_{H}$, is complete. Let us consider the space $\Gamma$ endowed with the Hausdorff metric $d_{H}$. It can be proved that also $\Gamma$ is complete (see [4], p. 90). Finally we recall that, whenever there exists a bounded set $Z \subset \mathbb{R}^{n}$ such that $K \subset Z$ for every $K \in \Gamma, \Gamma$ is a compact space.

For any $K \in \Gamma$, the inner vector optimization problem has the form

$$
\mathcal{V}-\max _{x \in K} f(x),
$$

where $\mathcal{V}$ - $\max _{x \in K}$ denotes the maximization with respect to the Pareto order $\leqq$ induced by the cone $\mathbb{R}_{+}^{m}$. The set-valued map $\Phi: \Gamma \rightrightarrows \mathbb{R}^{m}$ defined by

$$
\Phi(K):=\operatorname{Max} f(K)
$$

is well defined on $\Gamma$, i.e. $\operatorname{ArgMax}(f, K) \neq \varnothing$, for any $K \in \Gamma$. This follows from the compactness of $K$ (see, e.g., [18]). 
Remark 4.2 Since $f(K)$ is a compact set, by Remark 3.2, we have

$$
f(K) \subset \Phi(K)-\mathbb{R}_{+}^{m} .
$$

For the outer set-valued optimization problem

$$
\mathcal{S}-\min _{K \in \Gamma} \Phi(K) .
$$

we adopt the solution concept introduced in Definition 3.1.

We consider the minimax problem

$$
\mathcal{S}-\min _{K \in \Gamma} \mathcal{V}-\max _{x \in K} f(x) .
$$

In order to ensure the existence of a critical point in each set $K$ that solves the minimax problem we impose the following geometrical condition:

$$
\text { WMax } f(K) \subset\left(f\left(x_{1}\right)+\operatorname{int} \mathbb{R}_{+}^{m}\right) \cap\left(f\left(x_{2}\right)+\operatorname{int} \mathbb{R}_{+}^{m}\right) \text { for every } K \in \Gamma \text {. }
$$

Clearly, condition $(G)$ implies that

$$
\Phi(K) \subset\left(f\left(x_{1}\right)+\operatorname{int} \mathbb{R}_{+}^{m}\right) \cap\left(f\left(x_{2}\right)+\operatorname{int} \mathbb{R}_{+}^{m}\right) \text { for every } K \in \Gamma .
$$

If $f$ is a scalar-valued function, then $\Phi(K)=\max _{x \in K} f(x)$ and condition $(G)$ reduces to $\max _{x \in K} f(x)>\max \left\{f\left(x_{1}\right), f\left(x_{2}\right)\right\}$. This condition constitutes one of the main assumptions of mountain pass theorems for scalar-valued mappings, see e.g. [5, 26].

Now we prove the main result of this section that ensures the existence of a critical point of $f$ whenever a solution of problem $(\mathcal{O}-P)$ exists.

Theorem 4.3 Let $f \in \mathcal{C}^{1}\left(\mathbb{R}^{n}, \mathbb{R}^{m}\right)$ and the geometrical condition $(G)$ holds. Let $\hat{K} \in \Gamma$ be a solution of problem $(\mathcal{O}-P)$. Then there exists a critical point $c$ for $f$ such that

$$
f(c) \in \operatorname{cl}(\Phi(\hat{K})) .
$$

Proof By contradiction, let us suppose that every $x \in f^{-1}(\operatorname{cl}(\Phi(\hat{K})))$ is not a critical point for $f$. By Remark 2.4, $\|v(x)\|>0$ for every $x \in E$, where (check) $E=$ $f^{-1}(\operatorname{cl}(\Phi(\hat{K})))$. By Theorem 2.6 and by the compactness of the set E, there exist two positive real numbers $\delta$ and $\varepsilon$ such that

$$
\|v(x)\|>\delta,
$$

for every $x \in B_{\varepsilon}(E):=\left\{x \in \mathbb{R}^{n}: d(x, E)<\varepsilon\right\}$. By the geometrical condition $(G)$, we can choose the number $\varepsilon$ in such a way that $x_{1}, x_{2} \notin B_{\varepsilon}(E)$.

Now we consider the continuous path $\eta: \hat{K} \times[0,1] \rightarrow \mathbb{R}^{n}$ given by

$$
\eta(x, t)=x+t \psi(x) v(x)
$$

where $\psi: \hat{K} \rightarrow[0,1]$ is a continuous cut-off function such that

$$
\psi(x)=\left\{\begin{array}{cc}
0 & x \notin B_{\varepsilon}(E) \\
1 & x \in E
\end{array} .\right.
$$

Therefore

$$
\left.\frac{d}{d t} f_{i}(\eta(x, t))\right|_{t=0}=\psi(x)\left\langle\nabla f_{i}(x), v(x)\right\rangle \leq-\psi(x)\left(s_{f}(x)\right)^{2}
$$


for every $i=1, \ldots, m$, since $v(x) \in V_{f}(x)$. By Proposition 2.5, $s_{f}(x)=\|v(x)\|$. Hence

$$
\left.\frac{d}{d t} f_{i}(\eta(x, t))\right|_{t=0} \leq-\psi(x) \delta^{2}<0 .
$$

for every $x \in B_{\varepsilon}(E)$. Since $f \in \mathcal{C}^{1}, v$ is continuous and $\hat{K}$ is a compact set, there exists a real number $T>0$ such that for every $x \in B_{\varepsilon}(E)$

$$
\frac{d}{d t} f_{i}(\eta(x, t)) \leq-\frac{1}{2} \psi(x) \delta^{2}
$$

for every $t \in[0, T]$ and for every $i=1, \ldots, m$.

We consider the set $\eta(\hat{K}, T)$. Since $\eta\left(x_{1}, T\right)=x_{1}, \eta\left(x_{2}, T\right)=x_{2}$ and $\hat{K}$ is a compact and connected set, we obtain that $\eta(\hat{K}, T) \in \Gamma$. Now, for every $i=1, \ldots, m$ and for every $x \in B_{\varepsilon}(E)$

$$
f_{i}(\eta(x, T))=f_{i}(x)+\int_{0}^{T} \frac{d}{d t} f_{i}(\eta(x, t)) d t \leq f_{i}(x)-\frac{1}{2} T \delta^{2} .
$$

On the other hand

$$
x \in \hat{K} \backslash B_{\varepsilon}(E) \Longrightarrow f_{i}(\eta(x, T))=f_{i}(x) .
$$

Moreover, by (4), we have

$$
x \in B_{\varepsilon}(E) \Longrightarrow f_{i}(\eta(x, T)) \leq f_{i}(x)-\frac{1}{2} T \delta^{2}<f_{i}(x) .
$$

Hence we have proved that

$$
\begin{aligned}
& (\eta(\hat{K}, T)) \subset f(\hat{K})-\mathbb{R}_{+}^{m}, \\
& \Phi(\eta(\hat{K},)) \neq \Phi(\hat{K}) .
\end{aligned}
$$

By Remark 4.2, the domination property holds for every $K \in \Gamma$, i.e.,

$$
f(\hat{K}) \subset \Phi(\hat{K})-\mathbb{R}_{+}^{m} .
$$

Therefore, by (5) and (7), we obtain

$$
\Phi(\eta(\hat{K}, T)) \subset f(\eta(\hat{K}, T)) \subset f(\hat{K})-\mathbb{R}_{+}^{m} \subset \Phi(\hat{K})-\mathbb{R}_{+}^{m} .
$$

Hence

$$
\Phi(\eta(\hat{K}, T)) \subset \Phi(\hat{K})-\mathbb{R}_{+}^{m}
$$

Since, by assumption, $\hat{K}$ is a solution of the problem $(\mathcal{O}-P)$, inclusion $(8)$ implies that

$$
\Phi(\hat{K}) \subset \Phi(\eta(\hat{K}, T))-\mathbb{R}_{+}^{m}
$$

and consequently

$$
f(\hat{K}) \subset \Phi(\hat{K})-\mathbb{R}_{+}^{m} \subset \Phi(\eta(\hat{K}, T))-\mathbb{R}_{+}^{m} \subset f(\eta(\hat{K}, T))-\mathbb{R}_{+}^{m} .
$$

In view of Remark 3.2 the last inclusion together with (5) implies that $\Phi(\eta(\hat{K}, T))=$ $\Phi(\hat{K})$, which contradicts (6). 
Theorem 4.3 gives the existence of critical values $f(c)$ on the closures of the sets $\operatorname{Max} f(\hat{K})$ for every solution $\hat{K}$ to problem $(\mathcal{O}-P)$. Since $\hat{K}$ is compact and $f$ is continuous, $f(\hat{K})$ is a closed set. Hence, $\operatorname{cl}(\Phi(\hat{K}))=\operatorname{cl}(\operatorname{Max} f(\hat{K})) \subset \operatorname{WMax} f(\hat{K})$.

Remark 4.4 When we consider a $\mathcal{C}^{1}$ scalar-valued function $f: \mathbb{R}^{n} \longrightarrow \mathbb{R}$, we can compare Theorem 4.3 with the classical finite dimensional mountain pass theorem (see, e.g., Theorem 1.1 in [26] or, for a slightly different formulation, Theorem 2.1 in [5]). In this framework, our assumption on the existence of a solution $\hat{K}$ for the problem $(\mathcal{O}-P)$ is implied by the coercivity of $f$. Moreover, we underline that the assumption of the coercivity of $f$ implies that the geometric condition $(G)$ holds (see also the end of Section 6).

\section{Perturbed Minimax Problems}

The main aim of this section is to prove that every strict minimizer $\tilde{K}_{\varepsilon}$ (where $\varepsilon>$ $0)$ of the perturbed set-valued map $\Phi_{\varepsilon}(K)=\Phi(K)+\varepsilon d_{H}\left(K, \tilde{K}_{\varepsilon}\right) e$, where $\Phi(K):=$ Max $f(K)$, contains at least an almost critical point $x_{\varepsilon}$ for the function $f$ (i.e., a point $x_{\varepsilon}$ such that $s_{f}\left(x_{\varepsilon}\right)$ is close to zero). We underline that the idea of almost critical point is based on the function $s_{f}(x)$ introduced in Section 2.1 that can be interpreted as a sort of "distance from criticality". Moreover, we show that the almost critical point $x_{\varepsilon}$ is a counterimage of a weakly maximal point of the set $f\left(\tilde{K}_{\varepsilon}\right)$. We need a preliminary lemma concerning the continuity properties of the (weak) optimal value set-valued map of the vector optimization problem $(\mathcal{V}-P)$. We remark that the proof of this Lemma is a simple application of known stability results, widely studied in the theory of vector optimization.

Lemma 5.1 The set-valued map $\Theta: \Gamma \rightrightarrows \mathbb{R}^{m}$ defined by

$$
\Theta(K):=\operatorname{WMax} f(K),
$$

is upper semicontinuous on $\Gamma$ equipped with the Hausdorff metric.

Proof The thesis follows directly from Proposition 4.1 and Theorem 4.3, Ch. 4 in [18].

In order to prove the next result we introduce the following stronger geometrical condition: there exists a positive real number $\gamma$ such that

$$
\operatorname{WMax} f(K) \subset\left(f\left(x_{1}\right)+\gamma e+\mathbb{R}_{+}^{m}\right) \cap\left(f\left(x_{2}\right)+\gamma e+\mathbb{R}_{+}^{m}\right) \text { for every } K \in \Gamma,
$$

where $e=(1,1, \ldots, 1) \in \mathbb{R}_{+}^{m}$. Clearly, if condition $\left(G^{\prime}\right)$ holds for a given $\gamma$, then it holds for any $\tilde{\gamma}>0, \tilde{\gamma}<\gamma$, and moreover, it holds also for any $\kappa \in \operatorname{int} \mathbb{R}_{+}^{m}$, i.e. there exists $\gamma_{1}>0$ satisfying

$$
\operatorname{WMax} f(K) \subset\left(f\left(x_{1}\right)+\gamma_{1} \kappa+\mathbb{R}_{+}^{m}\right) \cap\left(f\left(x_{2}\right)+\gamma_{1} \kappa+\mathbb{R}_{+}^{m}\right) \text { for every } K \in \Gamma .
$$


Theorem 5.2 Let $0<\varepsilon<\frac{1}{2}$ and let the geometrical condition $\left(G^{\prime}\right)$ hold. Let $\tilde{K}_{\varepsilon} \in \Gamma$ be a strict minimizer for the set-valued map

$$
\Phi_{\varepsilon}(K)=\Phi(K)+\varepsilon d_{H}\left(K, \tilde{K}_{\varepsilon}\right) e,
$$

where, for any $K \in \Gamma$,

$$
d_{H}\left(K, \tilde{K}_{\varepsilon}\right)=\max \left\{\sup _{x \in K} \inf _{y \in \tilde{K}_{\varepsilon}}\|x-y\|, \sup _{y \in \tilde{K}_{\varepsilon}} \inf _{x \in K}\|x-y\|\right\} .
$$

Then there exists a point $x_{\varepsilon} \in \tilde{K}_{\varepsilon}$ such that $s_{f}\left(x_{\varepsilon}\right) \leq \sqrt{2 \varepsilon}$ and $f\left(x_{\varepsilon}\right) \in \operatorname{WMax} f\left(\tilde{K}_{\varepsilon}\right)$.

Proof For every $\delta>0$ consider the nonempty set

$$
\tilde{K}_{\varepsilon}^{\delta}=\left\{x \in \tilde{K}_{\varepsilon}:\left(f(x)+\delta e+\mathbb{R}_{+}^{m}\right) \cap f\left(\tilde{K}_{\varepsilon}\right)=\varnothing\right\} .
$$

Observe that we can choose $\delta>0$ such that $\tilde{K}_{\varepsilon}^{2 \delta} \cap\left\{x_{1}, x_{2}\right\}=\varnothing$. Indeed, by contradiction, if it were

$$
\left(f\left(x_{1}\right)+2 \delta e+\mathbb{R}_{+}^{m}\right) \cap f\left(\tilde{K}_{\varepsilon}\right)=\varnothing
$$

for every $\delta>0$, then it would be $\left(f\left(x_{1}\right)+2 \delta e+\mathbb{R}_{+}^{m}\right) \cap \operatorname{WMax} f\left(\tilde{K}_{\varepsilon}\right)=\varnothing$ contrary to the geometrical condition $\left(G^{\prime}\right)$. By the continuity of $f$, there exists $\bar{\delta}>0$ such that

$$
\tilde{K}_{\varepsilon}^{\delta} \cap\left(\left\{x_{1}, x_{2}\right\}+B_{\bar{\delta}}(0)\right)=\varnothing .
$$

By the continuity of $f$ and by the geometrical condition $\left(G^{\prime}\right)$, there exists $\rho>0$ such that for every $K \in \Gamma$

$$
f^{-1}(\mathrm{WMax} f(K)) \cap\left(\left\{x_{1}, x_{2}\right\}+B_{\rho}(0)\right)=\varnothing .
$$

Let $\sigma=\min \{\rho, \bar{\delta}\}$. There exists a continuous function $\psi: \tilde{K}_{\varepsilon} \rightarrow[0,1]$ such that

$$
\begin{gathered}
\psi(x)=1 \text { for every } x \in \tilde{K}_{\varepsilon}^{\delta}, \\
\frac{1}{2}<\psi(x) \leq 1 \text { for every } x \in \tilde{K}_{\varepsilon} \backslash\left(\left(x_{1}+B_{\sigma}(0)\right) \cup\left(x_{2}+B_{\sigma}(0)\right)\right), \\
0<\psi(x) \leq \frac{1}{2} \text { for every } x \in\left[\tilde{K}_{\varepsilon} \cap\left(\left(x_{1}+B_{\sigma}(0)\right) \cup\left(x_{2}+B_{\sigma}(0)\right)\right)\right] \backslash\left\{x_{1}, x_{2}\right\}
\end{gathered}
$$

and $\psi\left(x_{1}\right)=\psi\left(x_{2}\right)=0$. Now, we introduce a continuous function $h: \tilde{K}_{\varepsilon} \rightarrow \mathbb{R}^{n}$ defined as

$$
h(x)=\left\{\begin{array}{cl}
v(x) & \text { if }\|v(x)\| \leq 1 \\
\frac{v(x)}{s_{f}(x)} & \text { if }\|v(x)\|>1
\end{array}\right.
$$

where $v(x)$ is a vector pseudogradient defined by (3) in Section 2. Let us consider the function $\hat{h}: \tilde{K}_{\varepsilon} \rightarrow \mathbb{R}^{n}$,

$$
\hat{h}(x):=\psi(x) h(x)
$$


As a consequence of Proposition 2.5, $\|\hat{h}(x)\| \leq 1$ for every $x \in \tilde{K}_{\varepsilon}$. By Definition 2.3,

$$
\begin{array}{r}
{\left[f^{\prime}(x)\right] \hat{h}(x) \in-\psi(x)\left[s_{f}(x)\right]^{2} e-\mathbb{R}_{+}^{m} \quad \text { if }\|v(x)\| \leq 1} \\
{\left[f^{\prime}(x)\right] \hat{h}(x) \in-\psi(x) s_{f}(x) e-\mathbb{R}_{+}^{m} \quad \text { if }\|v(x)\|>1}
\end{array}
$$

Let us consider the deformation $\eta_{r}: \tilde{K}_{\varepsilon} \rightarrow \mathbb{R}^{n}, \eta_{r}(x)=x+r \hat{h}(x)$, where $r$ is a positive real number. By Theorem 2.6, the image $\hat{K}_{r}=\eta_{r}\left(\tilde{K}_{\varepsilon}\right)$ belongs to $\Gamma$, i.e. $\hat{K}_{r} \in \Gamma$. By Taylor's formula, for every $x \in \tilde{K}_{\varepsilon}$,

$$
f(x+r \hat{h}(x))-f(x)=r\left[f^{\prime}(x)\right] \hat{h}(x)+o(r) e .
$$

Since $\tilde{K}_{\varepsilon}$ is a strict minimizer of $\Phi_{\varepsilon}$, it holds

$$
\Phi\left(\hat{K}_{r}\right)+\varepsilon d_{H}\left(\tilde{K}_{\varepsilon}, \hat{K}_{r}\right) e \nsubseteq \Phi\left(\tilde{K}_{\varepsilon}\right)-\mathbb{R}_{+}^{m} .
$$

Therefore, a point $u_{r} \in f^{-1}\left(\Phi\left(\hat{K}_{r}\right)\right)$ exists such that

$$
f\left(u_{r}\right)+\varepsilon d_{H}\left(\tilde{K}_{\varepsilon}, \hat{K}_{r}\right) e \notin \Phi\left(\tilde{K}_{\varepsilon}\right)-\mathbb{R}_{+}^{m} .
$$

Clearly, there exists a point $x_{r}^{u} \in \tilde{K}_{\varepsilon}$ such that $f\left(x_{r}^{u}+r \hat{h}\left(x_{r}^{u}\right)\right)=f\left(u_{r}\right) \in \Phi\left(\hat{K}_{r}\right)$. By Remark 4.2, the domination property holds for $f\left(\tilde{K}_{\varepsilon}\right)$ and hence there exists $\tilde{x}_{r}^{u} \in$ $\Phi\left(\tilde{K}_{\varepsilon}\right)$ such that $f\left(\tilde{x}_{r}^{u}\right)-f\left(x_{r}^{u}\right) \in \mathbb{R}_{+}^{m}$. By (12), we get

$$
f\left(x_{r}^{u}+r \hat{h}\left(x_{r}^{u}\right)\right)-f\left(\tilde{x}_{r}^{u}\right) \in r\left[f^{\prime}\left(x_{r}^{u}\right)\right] \hat{h}\left(x_{r}^{u}\right)+o(r) e-\mathbb{R}_{+}^{m} .
$$

By (10), we have

$$
f\left(x_{r}^{u}+r \hat{h}\left(x_{r}^{u}\right)\right)-f\left(\tilde{x}_{r}^{u}\right) \in-r \psi\left(x_{r}^{u}\right)\left[s_{f}\left(x_{r}^{u}\right)\right]^{2} e+o(r) e-\mathbb{R}_{+}^{m} \quad \text { if }\left\|v\left(x_{r}^{u}\right)\right\| \leq 1
$$

By (11), we have

$$
f\left(x_{r}^{u}+r \hat{h}\left(x_{r}^{u}\right)\right)-f\left(\tilde{x}_{r}^{u}\right) \in-r \psi\left(x_{r}^{u}\right) s_{f}\left(x_{r}^{u}\right) e+o(r) e-\mathbb{R}_{+}^{m} \quad \text { if }\left\|v\left(x_{r}^{u}\right)\right\|>1
$$

By (13) it holds

$$
f\left(x_{r}^{u}+r \hat{h}\left(x_{r}^{u}\right)\right)-f\left(\tilde{x}_{r}^{u}\right) \notin-\varepsilon d_{H}\left(\tilde{K}_{\varepsilon}, \hat{K}_{r}\right) e-\mathbb{R}_{+}^{m},
$$

Combining (14) and (15) with (16), we get

$$
\begin{aligned}
& r \psi\left(x_{r}^{u}\right)\left[s_{f}\left(x_{r}^{u}\right)\right]^{2}+o(r) \leq \varepsilon d_{H}\left(\tilde{K}_{\varepsilon}, \hat{K}_{r}\right) \quad \text { if }\left\|v\left(x_{r}^{u}\right)\right\| \leq 1, \\
& r \psi\left(x_{r}^{u}\right) s_{f}\left(x_{r}^{u}\right)+o(r) \leq \varepsilon d_{H}\left(\tilde{K}_{\varepsilon}, \hat{K}_{r}\right) . \text { if }\left\|v\left(x_{r}^{u}\right)\right\|>1 .
\end{aligned}
$$

Now, let us consider a sequence $\left\{r_{n}\right\}$ of positive real numbers such that $r_{n} \rightarrow 0$ when $n \rightarrow+\infty$. By the compactness of $\tilde{K}_{\varepsilon}$, there exist a subsequence $\left\{x_{r_{n_{j}}}^{u}\right\}$ of $\left\{x_{r_{n}}^{u}\right\}$ and a point $x_{\varepsilon} \in \tilde{K}_{\varepsilon}$ such that $x_{r_{n_{j}}}^{u} \underset{j \rightarrow+\infty}{\rightarrow} x_{\varepsilon}$. By the continuity and the boundedness of the function $\hat{h}$ on $\tilde{K}_{\varepsilon}, r_{n_{j}} \hat{h}\left(x_{r_{n_{j}}}^{u}\right) \underset{j \rightarrow+\infty}{\rightarrow} 0$ and therefore

$$
y_{j}=x_{r_{n_{j}}}^{u}+r_{n_{j}} \hat{h}\left(x_{r_{n_{j}}}^{u}\right) \rightarrow x_{\varepsilon} \quad \text { as } j \rightarrow+\infty
$$


Since $f\left(y_{j}\right) \in \Phi\left(\hat{K}_{r_{n_{j}}}\right) \subset \mathrm{WMax} f\left(\hat{K}_{r_{n_{j}}}\right)$, and $\hat{K}_{r_{n_{j}}} \rightarrow \tilde{K}_{\varepsilon}$, we conclude by Lemma 5.1, that $f\left(x_{\varepsilon}\right) \in \mathrm{WMax} f\left(\tilde{K}_{\varepsilon}\right)$.

By the construction of the cut-off function $\psi$, there exists an integer $j_{0}$ such that

$$
\psi\left(x_{r_{n_{j}}}^{u}\right)>\frac{1}{2} \text { for every } j>j_{0} .
$$

Since $d_{H}\left(\tilde{K}_{\varepsilon}, \hat{K}_{r}\right) \leq r$, equation (17) and (18) gives

$$
\begin{gathered}
\frac{r_{n_{j}}}{2}\left[s_{f}\left(x_{r_{n_{j}}}^{u}\right)\right]^{2}+o\left(r_{n_{j}}\right) \leq \varepsilon d_{H}\left(\tilde{K}_{\varepsilon}, \hat{K}_{r_{n_{j}}}\right) \leq \varepsilon r_{n_{j}} \quad \text { if }\left\|v\left(x_{r_{n_{j}}}^{u}\right)\right\| \leq 1, \\
\frac{r_{n_{j}}}{2} s_{f}\left(x_{r_{n_{j}}}^{u}\right)+o\left(r_{n_{j}}\right) \leq \varepsilon d_{H}\left(\tilde{K}_{\varepsilon}, \hat{K}_{r_{n_{j}}}\right) \leq \varepsilon r_{n_{j}} . \quad \text { if }\left\|v\left(x_{r_{n_{j}}}^{u}\right)\right\|>1 .
\end{gathered}
$$

Therefore

$$
\begin{gathered}
{\left[s_{f}\left(x_{r_{n_{j}}}^{u}\right)\right]^{2}+\frac{o\left(r_{n_{j}}\right)}{r_{n_{j}}} \leq 2 \varepsilon \text { if }\left\|v\left(x_{r_{n_{j}}}^{u}\right)\right\| \leq 1,} \\
s_{f}\left(x_{r_{n_{j}}}^{u}\right)+\frac{o\left(r_{n_{j}}\right)}{r_{n_{j}}} \leq 2 \varepsilon \text { if }\left\|v\left(x_{r_{n_{j}}}^{u}\right)\right\|>1 .
\end{gathered}
$$

Since $\left\|v\left(x_{r_{n_{j}}}^{u}\right)\right\|=s_{f}\left(x_{r_{n_{j}}}^{u}\right)$ and $0<\varepsilon<1 / 2$, eventually (22) cannot hold. Finally, by letting $j \rightarrow+\infty$ in (21), in view of the continuity of the function $s_{f}$, we get $s_{f}\left(x_{\varepsilon}\right) \leq$ $\sqrt{2 \varepsilon}$ which completes the proof.

\section{A Minimax Theorem for Vector-valued Functions}

In this section we prove the main result of this paper. It is a finite dimensional version of a mountain pass theorem for vector-valued functions. In order to prove this theorem we need a lemma on the continuity properties of the set-valued map $\Phi$. We remark that the proof of this lemma is a direct application of the stability properties of a vector optimization problem.

Lemma 6.1 The set-valued map $\Phi: \Gamma \rightrightarrows \mathbb{R}^{m}$

$$
\Phi(K):=\operatorname{Max} f(K)
$$

is lower semicontinuous. Moreover, if the geometrical condition (G) holds, then $\Phi(\Gamma)=\bigcup_{K \in \Gamma} \Phi(K)$ is $\mathbb{R}_{+}^{m}$-bounded.

Proof The lower semicontinuity easily follows from Proposition 4.1 and Theorem 4.4, Ch. 4 in [18]. Moreover $\Phi(\Gamma)$ is $\mathbb{R}_{+}^{m}$-bounded by the geometric condition $(G)$.

In order to prove our results we introduce a notion that can be considered as a vector counterpart of the famous Palais-Smale condition. The following condition can be seen as a compactness requirement, since it ensures that a converging 
subsequence can be extracted from every sequence of almost critical points whose distance from criticality, measured according to the function $s_{f}(x)$, tends to zero.

Definition 6.2 (Vector Palais-Smale Condition) Let $\left\{x_{n}\right\}$ be a sequence such that

1. $\left\{f\left(x_{n}\right)\right\}$ is a $\mathbb{R}_{+}^{m}$-bounded sequence,

2. $s_{f}\left(x_{n}\right) \rightarrow 0$.

The function $f$ satisfies the Palais-Smale Condition (or, briefly the condition (PS)) if every sequence $\left\{x_{n}\right\}$ satisfying 1 . and 2 . has a convergent subsequence.

Remark 6.3 The Vector Palais-Smale Condition introduced in Definition 6.2 is a special case (for $\mathcal{C}^{1}$ single-valued, vector-valued functions $f$ ) of the two PalaisSmale-type conditions introduced in $[2,3]$ in the more general setting of set-valued maps. The condition in [2] is based on the notion of normal subdifferentials, whereas the condition in [3] is formulated with the help of Fréchet subdifferentials.

One should note that these two conditions as well as the Vector Palais-Smale Condition of Definition 6.2, when applied to scalar-valued functions, become slightly stronger than the original Palais-Smale condition (see, e.g., [26]). Namely, these conditions apply to sequences whose values are bounded from below, while the original Palais-Smale condition concerns sequences with bounded values. Some other generalizations of the original Palais-Smale condition to set-valued maps can be found in $[9,11]$, but they are too weak for our aims. The Palais-Smale-type conditions introduced in $[2,3]$ and in $[9,11]$ were used in proving the existence of solutions of set-valued optimization problems with a solution concept which differs from the solution concept considered in the present paper.

By the continuity of the function $s_{f}$ (see Section 2.1), it follows immediately that convergent subsequences of sequences $\left\{x_{n}\right\}$ satisfying the conditions 1 . and 2. of Definition 6.2 converge to a critical point for the function $f$. Hence, we use the variational principle of Theorem 3.5 and Theorem 5.2 to prove the existence of a sequence of almost critical points and next we use the Palais-Smale condition to ensure the existence of a subsequence converging to a critical point.

Theorem 6.4 (Mountain Pass Theorem) Let $f=\left(f_{1}, \ldots, f_{m}\right): \mathbb{R}^{n} \rightarrow \mathbb{R}^{m}$ be a function such that $f \in \mathcal{C}^{1}\left(\mathbb{R}^{n}, \mathbb{R}^{m}\right)$. Let the geometrical condition $\left(G^{\prime}\right)$ holds. If $f$ satisfies the condition (PS), then a critical point $c \in \mathbb{R}^{n}$ of $f$ exists.

Moreover, for any sequence $\left\{\varepsilon_{n}\right\}$ of positive real numbers converging to 0 there exists a sequence $\left\{x_{\varepsilon_{n}}\right\}$ such that

$$
c=\lim _{n \rightarrow+\infty} x_{\varepsilon_{n}}
$$

and $f\left(x_{\varepsilon_{n}}\right) \in \mathrm{WMax} f\left(\tilde{K}_{\varepsilon_{n}}\right)$ for every $n$, where $\tilde{K}_{\varepsilon_{n}} \in \Gamma$ is a strict minimizer of the setvalued map

$$
\Phi_{\varepsilon_{n}}(K)=\Phi(K)+\varepsilon_{n} d_{H}\left(K, \tilde{K}_{\varepsilon_{n}}\right) e,
$$

where $d_{H}\left(K, \tilde{K}_{\varepsilon_{n}}\right)$ is the Hausdorff distance, $e=(1,1, \ldots, 1) \in \mathbb{R}^{m}$ and $\Phi(K)=$ $\operatorname{Max} f(K)$. 
Proof It follows from Lemma 6.1 that the set-valued map $\Phi: \Gamma \rightrightarrows \mathbb{R}^{m}$ is lower semicontinuous and $\Phi(\Gamma)$ is $\mathbb{R}_{+}^{m}$-bounded.

Let $\left\{\varepsilon_{n}\right\} \subset \mathbb{R}, \varepsilon_{n}>0, \varepsilon_{n} \rightarrow 0$. By Theorem 3.5 , there exists a sequence of sets $\left\{\tilde{K}_{\varepsilon_{n}}\right\}, \tilde{K}_{\varepsilon_{n}} \in \Gamma$ such that for each $n \in \mathbb{N}$ the set $\tilde{K}_{\varepsilon_{n}}$ is a strict minimizer of $\Phi_{\varepsilon_{n}}(K)$ $n \in \mathbb{N}$.

By Theorem 5.2, there exists a sequence $\left\{x_{\varepsilon_{n}}\right\}, x_{\varepsilon_{n}} \in \tilde{K}_{\varepsilon_{n}}$, such that $s_{f}\left(x_{\varepsilon}\right) \leq \sqrt{2 \varepsilon}$. Hence, $s_{f}\left(x_{\varepsilon_{n}}\right) \rightarrow 0$ when $n \rightarrow+\infty$. Therefore, since $f\left(x_{\varepsilon_{n}}\right) \in \mathrm{WMax} f\left(\tilde{K}_{\varepsilon_{n}}\right)$, by the geometrical condition $\left(G^{\prime}\right),\left\{f\left(x_{\varepsilon_{n}}\right)\right\}$ is a $\mathbb{R}_{+}^{m}$-bounded sequence. Now the condition (PS) ensures that $\left\{x_{\varepsilon_{n}}\right\}$ has a convergent subsequence $\left\{x_{\varepsilon_{n_{k}}}\right\}$ such that $x_{\varepsilon_{n_{k}}} \rightarrow c$.

In the corollary below we use the following uniform boundedness condition. We say that the family of sets $\Gamma$ satisfies the uniform boundedness condition if there exists a bounded set $Z$ such that $K \subset Z$ for every $K \in \Gamma$.

With the help of the uniform boundedness condition we can obtain a minimax characterization of critical points $c$ the existence of which is proved in Theorem 6.4.

Corollary 6.5 Let $f=\left(f_{1}, \ldots, f_{m}\right): \mathbb{R}^{n} \rightarrow \mathbb{R}^{m}$ be a function of class $C^{1}, f \in \mathcal{C}^{1}\left(\mathbb{R}^{n}\right.$, $\left.\mathbb{R}^{m}\right)$. Let the geometrical condition $\left(G^{\prime}\right)$ hold. Under the assumption of the uniform boundedness condition for the family $\Gamma$, there exists a critical point $\bar{x} \in \mathbb{R}^{n}$ for the function $f$. Moreover, there exists $\bar{K} \in \Gamma$ such that $\bar{K}$ is a solution of the set-valued optimization problem $(\mathcal{O}-P)$ and

$$
f(c) \in \operatorname{WMax} f(\bar{K}) .
$$

Proof The uniform boundedness of the family $\Gamma$ implies that the Palais-Smale condition (PS) holds. In Theorem 6.4 the existence of a converging sequence $\left\{x_{\varepsilon_{n}}\right\}$ of points such that $f\left(x_{\varepsilon_{n}}\right) \in \mathrm{WMax} f\left(\tilde{K}_{\varepsilon_{n}}\right)$, where $\tilde{K}_{\varepsilon_{n}} \in \Gamma$ is a strict minimizer of the set-valued map

$$
\Phi_{\varepsilon_{n}}(K)=\Phi(K)+\varepsilon_{n} d_{H}\left(K, \tilde{K}_{\varepsilon_{n}}\right) e,
$$

is proved. Let $c \in \mathbb{R}^{n}$ be the critical point such that $x_{\varepsilon_{n}} \rightarrow c$. By Remark 4.1, $\Gamma$ endowed with the Hausdorff metric $d_{H}$ is a compact metric space. Consequently, the sequence $\left\{\tilde{K}_{\varepsilon_{n}}\right\}$ contains a subsequence $\left\{\tilde{K}_{\varepsilon_{n_{j}}}\right\} \subset \Gamma$ such that there exists $\bar{K} \in \Gamma$ satisfying

$$
d_{H}\left(\tilde{K}_{\varepsilon_{n_{j}}}, \bar{K}\right) \longrightarrow 0 \quad \text { as } j \rightarrow+\infty
$$

By Lemma 5.1, $f(c) \in \mathrm{WMax} f(\bar{K})$.

To complete the proof, we show that $\bar{K}$ is a strict solution to problem $(\mathcal{O}-P)$, i.e

$$
\Phi(K) \nsubseteq \Phi(\bar{K})-\mathbb{R}_{+}^{m}
$$

for every $K \in \Gamma, K \neq \bar{K}$.

Suppose on the contrary that there exists a set $K \in \Gamma, K \neq \bar{K}$, such that for every $z \in \Phi(K)$ one can find $\bar{z} \in \Phi(\bar{K})$ and $r_{z} \in \mathbb{R}_{+}^{m}$ satisfying

$$
z=\bar{z}-r_{z}
$$

Fix an $\ell \in \mathbb{N}$ and notice that there exists a $\kappa>0$ such that $\kappa B(0,1) \subseteq-e+\mathbb{R}_{+}^{m}$. 
By Lemma 6.1, the set-valued map $\Phi$ is lower semicontinuous at $\bar{K}$. This means that there exist $j_{\ell} \geq \ell$ and $z_{n_{j_{\ell}}} \in \Phi\left(K_{n_{j_{\ell}}}\right)$ such that

$$
z \in z_{n_{j_{\ell}}}-\varepsilon_{n_{\ell}} d_{H}\left(K, \tilde{K}_{n_{\ell}}\right) e+r_{n_{j_{\ell}}}-\mathbb{R}_{+}^{m} .
$$

Therefore,

$$
z+\varepsilon_{n_{j_{\ell}}} d_{H}\left(K, \tilde{K}_{n_{j_{\ell}}}\right) e \in z_{n_{j_{\ell}}}-\varepsilon_{n_{\ell}} d_{H}\left(K, \tilde{K}_{n_{\ell}}\right) e+\varepsilon_{n_{j_{\ell}}} d_{H}\left(K, \tilde{K}_{n_{j_{\ell}}}\right) e+r_{n_{j_{\ell}}}-\mathbb{R}_{+}^{m} .
$$

Since the sequences $\left\{\varepsilon_{n_{j_{\ell}}} d_{H}\left(K, \tilde{K}_{n_{j_{\ell}}}\right)\right\}$ and $\left\{r_{n_{j_{\ell}}}\right\}$ tend to zero we can choose $j_{\ell}$ sufficiently large so as to have

$$
\varepsilon_{n_{j_{\ell}}} d_{H}\left(K, \tilde{K}_{n_{j_{\ell}}}\right) e+r_{n_{j_{\ell}}}-\varepsilon_{n_{\ell}} d_{H}\left(K, \tilde{K}_{n_{\ell}}\right) e \in-\mathbb{R}_{+}^{m}
$$

And consequently

$$
z+\varepsilon_{n_{j_{\ell}}} d_{H}\left(K, \tilde{K}_{n_{j_{\ell}}}\right) e \in z_{n_{j_{\ell}}}-\mathbb{R}_{+}^{m}
$$

which contradicts the fact that for every $K \in \Gamma$ and for every $j \in \mathbb{N}$ we have

$$
\Phi(K)+\varepsilon_{n_{j}} d_{H}\left(K, \tilde{K}_{\varepsilon_{n_{j}}}\right) e \nsubseteq \Phi\left(\tilde{K}_{\varepsilon_{n_{j}}}\right)-\mathbb{R}_{+}^{m} .
$$

In this way we proved that $\bar{K}$ is a a strict solution to problem $(\mathcal{O}-P)$ which completes the proof.

Hence we locate a critical point as a minimax point in the sense that it is a weakly maximal solution of the inner problem $\left(\mathcal{I}-P_{K}\right)$, where $K=\bar{K}$ is the solution of the outer problem $(\mathcal{O}-P)$.

Whenever $f$ is a scalar-valued function, $f: \mathbb{R}^{n} \longrightarrow \mathbb{R}$, Corollary 6.5 reduces to the well known finite dimensional "mountain pass theorem" (see, e.g., [5] or [26]). Here we recall the result as stated in [5].

Theorem 6.6 Let $f \in \mathcal{C}^{1}\left(\mathbb{R}^{n}, \mathbb{R}\right)$ and coercive (i.e. $\left.\lim _{\|x\| \rightarrow+\infty} f(x)=+\infty\right)$. Suppose there are two points $x_{1}, x_{2}$ and a set $R$ disconnecting them, such that

$$
\inf _{R} f>\max \left\{f\left(x_{1}\right), f\left(x_{2}\right)\right\} .
$$

Then $f$ has a critical point $x_{3}$ (different from $x_{1}, x_{2}$ ). The critical value $c=f\left(x_{3}\right)$ can be characterized as follows: let

$$
\Gamma=\left\{K \subset \mathbb{R}^{n}: K \text { is compact, connected and contains } x_{1}, x_{2}\right\},
$$

then

$$
c=\inf _{K \in \Gamma} \max _{x \in K} f(x) .
$$

Let us note that for scalar-valued functions $f$ the geometrical condition $\left(G^{\prime}\right)$ takes the form: there exists a positive real number $\gamma$ such that

$$
\max _{x \in K} f(x)>\max \left\{f\left(x_{1}\right), f\left(x_{2}\right)\right\}+\gamma \text { for all } K \in \Gamma .
$$

If the scalar-valued function $f$ is coercive there exists a bounded set $Z$ such that for every set $K \in \Gamma$ with $K \nsubseteq Z$ the condition $\left(G^{\prime}\right)$ holds automatically. Therefore, we focus our attention on the family $\Gamma_{b}$ of sets $K \in \Gamma$ such that $K \subseteq Z$. Condition 
(25) implies that $\left(G^{\prime}\right)$ holds. Corollary 6.5 gives the thesis of Theorem 6.6 since $\inf _{K \in \Gamma} \max _{x \in K} f(x)=\inf _{K \in \Gamma_{b}} \max _{x \in K} f(x)=\max _{x \in \bar{K}} f(x)$, where $\bar{K}$ is the minimizer of the function from $\Gamma_{b}$ to $\mathbb{R}$ defined by $K \mapsto \max _{x \in K} f(x)$.

Acknowledgements The authors gratefully acknowledge the hospitality and the support of the Dipartimento di Economia, Università dell'Insubria, the Systems Research Institute of the Polish Academy of Sciences and the Cardinal Stefan Wyszynski University.

\section{References}

1. Aubin, J.P., Cellina, A.: Differential Inclusions. Springer, Berlin Heidelberg (1984)

2. Bao, T.Q., Mordukhovich, B.S.: Variational principles for set-valued mappings with applications to multiobjective optimization. Control Cybern. 36, 531-562 (2007)

3. Bao, T.Q., Mordukhovich, B.S.: Relative Pareto minimizers for multiobjective problems: existence and optimality conditions. Math. Program. 122(2, Ser. A) 301-347 (2010)

4. Beer, G.: Topologies on Closed and Closed Convex Sets. Kluwer, Dordrecht (1993)

5. Conti, M., Lucchetti, R.: The minimax approach to the critical point theory. In: Recent Developments in Well-Posed Variational Problems, pp. 29-76. Kluwer, Dordrecht (1995)

6. Degiovanni, M., Lucchetti, R., Ribarska, N.: Critical point theory for vector valued functions. J. Convex Anal. 9, 415-428 (2002)

7. Dontchev A.L., Zolezzi, T.: Well-Posed Optimization Problems. Springer (1993)

8. Göpfert, A., Riahi, H., Tammer, C., Zălinescu, C.: Variational Methods in Partially Ordered Spaces. Springer, New York (2003)

9. Ha, T.X.D.: The Ekeland variational principle for set-valued maps involving coderivatives. J. Math. Anal. Appl. 286, 509-523 (2003)

10. Ha, T.X.D.: Some variants of the Ekeland variational principle for a set-valued map. J. Optim. Theory Appl. 124, 187-206 (2005)

11. Ha, T.X.D.: Variants of the Ekeland variational principle for a set-valued map involving the Clarke normal cone. J. Math. Anal. Appl. 316, 346-356 (2006)

12. Huang, X.X.: New stability results for Ekeland's $\epsilon$-variational principles for vector-valued and set-valued maps. J. Math. Anal. Appl. 262, 12-23 (2001)

13. Huang, X.X.: A new variant of Ekeland's variational principle for set-valued maps. Optimization 52, 53-63 (2003)

14. Jabri, Y.: The Mountain Pass Theorem. Cambridge University Press, Cambridge (2004)

15. Jahn, J.: Vector Optimization. Theory, Applications and Extensions. Springer, Berlin (2004)

16. Klein, E., Thompson, A.C.: Theory of Correspondences. Wiley, New York (1984)

17. Kuroiwa, D.: On set-valued optimization. Nonlinear Anal. 47, 1395-1400 (2001)

18. Luc, D.T.: Theory of Vector Optimization. Springer (1989)

19. Lucchetti, R.E., Revalski, J.P., Théra, M.: Critical points for vector-valued functions. Control Cybern. 31, 545-555 (2002)

20. Miglierina, E.: Slow solutions of a differential inclusion and vector optimization. Set-Valued Anal. 12, 345-356 (2004)

21. Miglierina, E., Molho, E., Rocca; M.: Critical points index for vector functions and vector optimization. J. Optim. Theory Appl. 138, 479-496 (2008)

22. Smale, S.: Global analysis and economics. I. Pareto optimum and a generalization of Morse theory. In: Dynamical Systems (Proc. Sympos., Univ. Bahia, Salvador, 1971), pp. 531-544. Academic, New York (1973)

23. Smale, S.: Global analysis and economics. J. Math. Econ. 1, Part IIA: 1-14; Part III: 107-117; Part IV, 119-127; Part V: 213-221 (1974)

24. Smale, S.: Optimizing several functions. In: Manifolds-Tokyo 1973 (Proceedings of International Conference on Manifolds and Related Topics in Topology), pp. 69-75. University Tokyo Press, Tokyo (1975)

25. Smale, S.: Global analysis and economics VI. J. Math. Econ. 3, 1-14 (1976)

26. Struwe, M.: Variational Methods, 4th edn. Springer, Berlin, Heidelberg (2008) 